\title{
A Hybrid Method for the Cross-Cultural Adaptation of Self-Report Measures
}

\author{
Atul Kumar Goyal ${ }^{1}$ (D) - Jaimanti Bakshi ${ }^{1} \cdot$ Naresh K Panda $^{1} \cdot$ Rakesh Kapoor $^{2}$. \\ Dharam Vir ${ }^{1} \cdot$ Krishan Kumar $^{3} \cdot$ Pankaj Aneja $^{4}$ • Balwinder Singh ${ }^{5}$. \\ Meenu Gupta ${ }^{6} \cdot$ Sumittar Singh Walia $^{7}$
}

Accepted: 9 May 2020 / Published online: 21 May 2020

(C) Springer Nature Switzerland AG 2020

\begin{abstract}
Cross-cultural adaptation of self-reported measures to other languages endeavors to enhance the validity and reliability of the instrument for the local population. Traditionally, qualified academicians or language experts performed the translation task, but nowadays, various online translation tools are available for the same. The present study intends to compare the validity of traditional and online modes of translation of selfreported measures, and to develop a hybrid method by combining the traditional and online methods to achieve an accurate translation cost-effectively. For this purpose, the existing English version of shame and stigma scale was translated into the Hindi language traditionally by language experts, Google translator, and by a hybrid method. The validity of translated versions was tested using the content validity index (CVI). The CVI score was found to be 0.94 for the traditional method, which is significantly higher compared to the online translation method in which the CVI score was 0.42 . CVI score in the hybrid method comes to be 0.84 . The present study suggests that the online translation method alone is insufficient for the accurate translation of questionnaires and, therefore, it is recommended that hybrid method should be followed for the process of cross-cultural adaptation of self-report measures to impart high accuracy in a cost-effective manner.
\end{abstract}

Keywords Translation $\cdot$ Validation $\cdot$ Reliability $\cdot$ Questionnaire $\cdot$ Shame and stigma

\section{Introduction}

Despite considerable advancement in medical technologies, self-report measures remain the first choice for assessment of clinical symptoms, neurological, and various

Atul Kumar Goyal

atlgyl@gmail.com

Extended author information available on the last page of the article 
psychological disorders (Minden et al. 2014; Goyal and Saini 2019; Goyal 2015). Questionnaires provide various advantages like being cost-effective, rapid, and timesaving, high availability, and need less expertise for administration (Jones et al. 2013). However, compliance with local demographic conditions, including the language and culture of the area, is necessary while administration of the instrument; otherwise, there are high chances of false-positive or true negative results (Bolarinwa 2015). Most of the self-report measures are available in multiple languages. Cross-cultural adaptation of self-report measures to languages other than original is performed to enhance the validity, reliability, applicability, and generalization of the instrument (Tsang et al. 2017). Traditionally, qualified academicians from research institutes or language experts from state universities tend to perform the translation task. But with the advancement of technology, nowadays, various online translation tools are available to perform the task of translation (Balk et al. 2013). Google translator, developed by Google Inc. in 2006, is the most popular translation tool which offers translation between 103 languages (Mondal et al. 2019). Earlier, Google translator uses the predictive algorithm, which translates a sentence word by word leading to high grammatical inaccuracy. In 2016, Google translator introduced the neural machine algorithm, which translates the whole sentence at once, leading to high grammatical accuracy (Pathak and Pakray 2019; Guo 2016). Researchers around the world now prefer online translation to be cost-effective, readily available easy, and fast (Kirchhoff et al. 2011).

Recently English version of the Kessler psychological distress scale has translated into the Bengali language using Google translator and tested the validity of the online translated version by comparing the results with traditional translation. The authors have reported a high error rate, and lower equivalence in the online translated version compare to the traditional method of translation (Mondal et al. 2019). Chen et al. attempted to translate diabetes education study material into Spanish and Chinese language and found that Google translation is more accurate for English to Spanish than English to Chinese (Chen et al. 2016). Various challenges tends to occurs when an online translator is used for translation because the questionnaires are generally not developed with the intention to translate into other languages. Translation to other languages often leads to a lack of cultural equivalency due to complications offered by complex sentences, grammatical structure and syntax variations (Guo 2016). Most of the studies conducted so far which attempt to translate a questionnaire using an online translator reported the results to be skewed towards unsatisfied translations. However, it is not to be omitted that online translation can save a lot of time and resources. Therefore, in the present study, we propose a hybrid method in which the questionnaire is first translated using an online translator and then can be corrected manually by the traditional method.

In the present study, we took the shame and stigma scale to be tested for a hybrid translation method. Shame and stigma scale was first introduced by the Kissane et al. for the assessment of mutilation in patients of head neck cancer. The shame and stigma scale has 20 questions rated 0 to 4 on the Likert scale. A high value of scale indicates high shame and stigma in the patients (Kissane et al. 2013). Pirola et al. translated the shame and stigma scale into Portuguese (Brazil) and evaluated its reliability and validity (Pirola et al. 2017). Recently, Tseng et al. translated and validated the shame and stigma scale into the Chinese language (Tseng et al. 2019). In the present study, we intend to compare the validity of shame and stigma scale when translated in the Hindi 
language traditionally by language experts and when translated using online translator; and to develop a hybrid method which combines both online and traditional methods to carry out translation of a self-reported measure in a cost and time-effective manner.

\section{Methodology}

Shame and Stigma Scale This instrument consists of 20-items among which 8 items explain the shame at the appearance, 5 items measure the social avoidance, 4 items assess the sense of avoiding by society (stigma), and 3 items mentioned the general regret a person feels due to their past activities. 5-point Likert scale is used to obtain responses from participants. The value of the Likert scale range from 0 to 4 , indicating the "never" to "all the time" respectively. Shame and stigma scale reported to exhibit optimum internal validity and convergent validity when tested with the FACT-G, FACT-H\&N, PHQ questionnaire; and exhibit divergent validity with the social desirability scale (Kissane et al. 2013).

Online Translation Method For online translation, Google translator was used. The translation process was carried out in two phases, as described in previous studies (Mondal et al. 2019).

Forward Translation: Sentence from the original English version of the questionnaire was copied and pasted to the translation window provided by the web portal of Google translator. The translated language is then chosen as Hindi. The translated text from the web portal of Google was copied and pasted in a word document.

Reverse Translation: The translated Hindi text of the questionnaire is copied from a word document and pasted in the translation window of Google translator. The language of translation was chosen as English. The translated text is copied from the translation window and pasted in a word document.

Traditional Translation Method Traditional translation was carried using modified guidelines defined in previous studies (Beaton et al. 2000; Tseng et al. 2019). The translation process was conducted into two phases:

Forward translation: The translation of the original English version to the Hindi version was performed by two experts having a doctorate in language and currently holding a permanent academic position in the government institutes. The synthesis of translations was then carried out by the translators and researchers together to finalize the Hindi version of the instrument.

Reverse translation: The reverse translation of the Hindi version back to the English version was performed by two experts having a doctorate in language and currently holding a permanent academic position in the government institutes. The synthesis of translations was then carried out by the translators and researchers together to finalize the back-translated English version of the instrument.

Hybrid Translation Method For both forward and backward translation of scale, the online translation method is followed for initial translation, and then the online translated version is corrected by the traditional method (Fig. 1). 


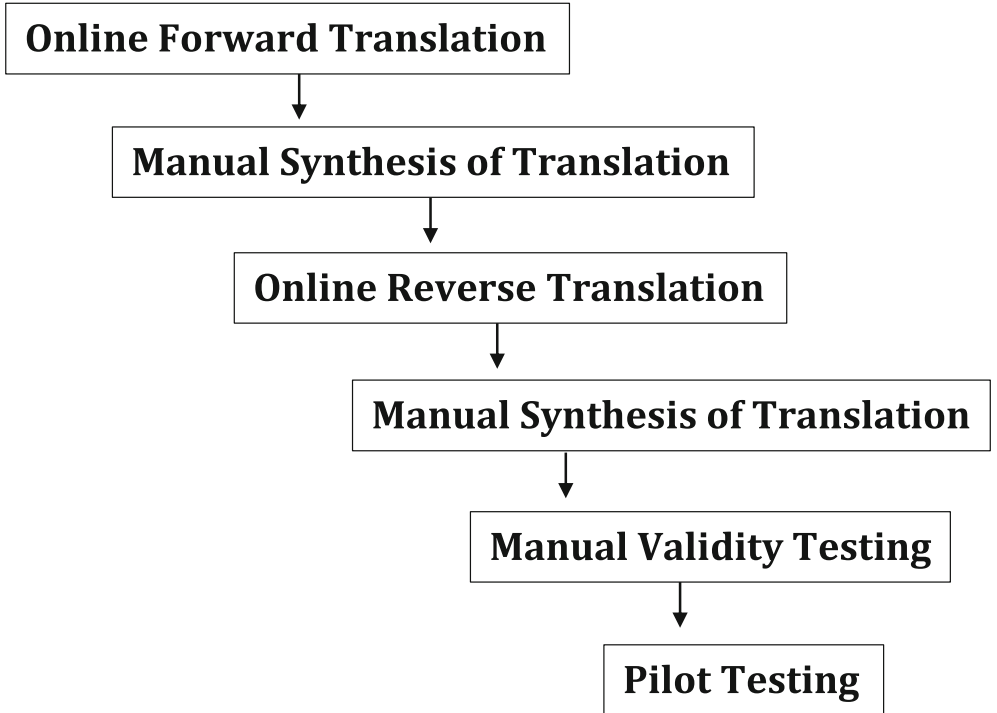

Fig. 1 Flow chart showing the Hybrid method for the cross-cultural adaptation of a self-reported measure, Hybrid method is a six-step approach in which the first four steps involve the online forward and backward translation and manual synthesis of online translation, and the last two steps include the manual validity testing and piolet testing of the translated scale

Validity Testing The committee of five experts which include the experts of language, psychologist and medical professional; analyzed all the versions of the questionnaire including the original English version, translated Hindi version and back-translated English versions; to check the language, conceptual aspect, semantic-idiomatic, and cultural parameters of the language using the content validity index (CVI) (Beaton et al. 2000; Tsang et al. 2017).

Content Validity Index The developed Hindi version will be validated using the face validity performed through the content validity index (CVI). In CVI, all five experts were asked to rate each of the translated items between 1 to 4 based on relevance, clarity, simplicity, and accuracy. The number of the agreement is then calculated by taking all the ratings score more than or equal to three. The CVI value for each item is then computed by dividing the number of ratings greater than or equal to 3 by the number of raters. For instance, a score of 0.80 indicates that four out of five experts rated a respective item greater than or equal to 3 regarding relevance, clarity, simplicity, and accuracy. The final CVI is then calculated by taking the average of all individual CVI. The final score of more than 0.75 will be considered as optimum for finalizing the scale (Yaghmaie 2003; Waltz and Bausell 1981).

\section{Results}

CVI score of the translated Hindi version of the instrument came to be 0.94 for the traditional translation method which is significantly higher compared to the CVI score of the online translation method which was 0.42 . In the hybrid method, the value of the 
CVI score comes to be 0.84 (Table 1). Two items () showed the identical score in online, traditional, and hybrid methods; three items showed a mild difference (Guo 2016; Prakash and Pallepati 2016; Rodrigues et al. 2017), whereas the rest of the items showed a moderate difference in score.

\section{Discussion}

Cross-cultural adaptation of self-report measures to other languages from the original language is considered as an academic endeavor that aims towards enhancing the reliability and validity of the instrument. Translation adaptation to local languages helps in eliminating the researcher's biases by making the instrument self-administrable by the patients (Beaton et al. 2000). The translation process may lead to losing reliability and validity of the instrument due to the modification of the logical meaning of the sentence. Therefore, it is necessary to validate the instrument to reach

Table 1 Table showing the content validity index (CVI) score obtained in the online, traditional, and hybrid translation methods. Highest CVI score $(0.94 \pm 0.09)$ was obtained in traditional method which indicates effective translation, whereas the lowest score $(0.42 \pm 0.27)$ was online method which indicates ineffective translation. Optimum score $(0.84 \pm 0.15)$ was obtained in hybrid method indicating acceptable translation

\begin{tabular}{llll}
\hline Item Number & \multicolumn{2}{l}{ CVI Score } & \\
\cline { 2 - 4 } & Online method & Traditional method & Hybrid method \\
\hline SSS-1 & 0.4 & 1 & 1 \\
SSS-2 & 0.4 & 0.8 & 0.6 \\
SSS-3 & 0.6 & 1 & 0.8 \\
SSS-4 & 0.6 & 1 & 1 \\
SSS-5 & 1 & 1 & 1 \\
SSS-6 & 0 & 1 & 0.8 \\
SSS-7 & 0.2 & 1 & 0.8 \\
SSS-8 & 0.4 & 1 & 1 \\
SSS-9 & 0.6 & 0.8 & 0.8 \\
SSS-10 & 0.2 & 0.8 & 0.6 \\
SSS-11 & 0.2 & 1 & 1 \\
SSS-12 & 0.2 & 1 & 0.6 \\
SSS-13 & 0 & 1 & 0.8 \\
SSS-14 & 0.2 & 0.8 & 0.8 \\
SSS-15 & 0.6 & 1 & 1 \\
SSS-16 & 0.4 & 1 & 1 \\
SSS-17 & 0.2 & 1 & 0.8 \\
SSS-18 & 0.8 & 1 & 1 \\
SSS-19 & 0.6 & 0.8 & 0.6 \\
SSS-20 & 0.8 & 0.8 & 0.8 \\
Mean CVI Score \pm SD & $0.42 \pm 0.27$ & $0.94 \pm 0.09$ & $0.84 \pm 0.15$ \\
\hline & & & \\
\hline
\end{tabular}


equivalence between the source and target versions of the questionnaire (Beaton et al. 2000). The task of translating and validating a questionnaire to other languages demands great investment of the time and effort to ensure psychometric properties are not altered. Apart from this, the translation process demand additional financial cost which is to be payable to the language experts who performed the translation task (Tsang et al. 2017). In the present study, with the aim towards developing a costeffective hybrid approach for the translation of a questionnaire to other languages, we compared the validity of the translation of shame and stigma scale when it was translated by traditional, online and hybrid method. We found that traditional translation method has considerably high validity compared to online translation. The findings showed that only three out of the 20 items exceeded the cut-off score for acceptable validity for the online translation method. Although the hybrid method yields less validity compare to the traditional method but yet showed validity to a satisfactory level in a cost-effective and time-saving manner.

Similar results were obtained by the previous study in which a high subjective sentence error rate was observed when the Kessler psychological distress scale is translated from the English language to the Bengali language (Mondal et al. 2019). Taylor et al. translated the strengths and difficulties questionnaire from 24 languages to English and Spanish using Babylon and Google translator and found that only 6 out of 48 translations were acceptable (Taylor et al. 2015). Guo also found that traditional translation is more accurate than the Google translation when pain care quality survey was translated into Mandarin language by both traditional and online method (Guo 2016). Vidhayasai et al. also reported that Google translation leads to an imperfect translation which can create major problems (Vidhayasai et al. 2015). Dhakar et al. reported that the Bing Translator performed better than Google translator while translating text, English to Hindi language or vice versa (Dhakar et al. 2013) (Table 2). Prakash and Pallepati validate the Kannada translated modified service quality questionnaire by using the content validity index (Prakash and Pallepati 2016). The CVI score achieved in their study was 0.96, which is similar to what we achieve in the present study using the traditional method. Lau et al. achieved a similar CVI score (0.97) during the translation of questionnaire to assess the gastrointestinal and upper respiratory tract related illnesses in pre-school children from the English language to Chinese language (Lau et al. 2018). Rodrigues et al. develop the personalized exercise questionnaire and tested its validity using the CVI (Rodrigues et al. 2017). So far only three studies have been conducted on shame and stigma for head and neck cancer. The first study was conducted by Kissane et al. authors developed the scale (Kissane et al. 2013), the second study was conducted by Pirola et al. in which authors translated the scale in Brazil language (Pirola et al. 2017), and the third study was conducted by Tseng et al. in which authors translated the scale into the Chinese language (Tseng et al. 2019).

As a strength, the present study is the first in which the shame and stigma scale is translated and validated into the Hindi language using online, traditional and hybrid methods. The only limitation of the present study is that we tested the feasibility of translation of a single scale in a single language using a single online translator and single validity tool. A more triangulate approach using multiple methods can give a more accurate idea about the feasibility of online translation. In present study we use the shame and stigma scale, further studies which could be based on translation of two or more scale can enhance generalizability in the hybrid method. Future studies which 
Table 2 Table showing the various studies which has reported use of an online translator (Google or Bing translator) for the cross-cultural language translation of a self-reported measure or any other text material

\begin{tabular}{|c|c|c|c|c|}
\hline S. No. & $\begin{array}{l}\text { Name of the } \\
\text { questionnaire/ } \\
\text { Material }\end{array}$ & Translation language & Important findings & Citation \\
\hline 1 & $\begin{array}{l}\text { Kessler } \\
\text { psychological } \\
\text { distress scale }\end{array}$ & $\begin{array}{l}\text { English to Bengali } \\
\text { language }\end{array}$ & $\begin{array}{l}\text { High error rate, and lower } \\
\text { equivalence in the online } \\
\text { method compare to the } \\
\text { traditional method }\end{array}$ & $\begin{array}{l}\text { Mondal et al. } \\
\text { (2019) }\end{array}$ \\
\hline 2 & $\begin{array}{l}\text { Diabetes education } \\
\text { study material }\end{array}$ & $\begin{array}{l}\text { English to Spanish and } \\
\text { Chinese language }\end{array}$ & $\begin{array}{l}\text { Google translation is more } \\
\text { accurate for Spanish than } \\
\text { to Chinese }\end{array}$ & Chen et al. (2016) \\
\hline 3 & $\begin{array}{l}\text { Pain Care Quality } \\
\text { Survey }\end{array}$ & $\begin{array}{l}\text { English to Mandarin } \\
\text { language }\end{array}$ & $\begin{array}{c}\text { Human translations showed } \\
\text { better translation quality } \\
\text { than Google Translation }\end{array}$ & Guo (2016) \\
\hline 4 & $\begin{array}{l}\text { Strengths and } \\
\text { difficulties } \\
\text { questionnaire }\end{array}$ & $\begin{array}{l}24 \text { languages to English } \\
\text { and Spanish } \\
\text { language }\end{array}$ & $\begin{array}{l}\text { Only } 6 \text { out of } 48 \text { Babylon and } \\
\text { Google translations were } \\
\text { acceptable }\end{array}$ & Taylor et al. (2015) \\
\hline 5 & $\begin{array}{l}\text { Terms and } \\
\text { Conditions } \\
\text { of an Airline }\end{array}$ & $\begin{array}{l}\text { English to Thai } \\
\text { language }\end{array}$ & $\begin{array}{l}\text { Google translation leads to an } \\
\text { imperfect translation } \\
\text { which can create major } \\
\text { problems }\end{array}$ & $\begin{array}{l}\text { Vidhayasai } \\
\text { et al. (2015) }\end{array}$ \\
\hline 6 & $\begin{array}{l}\text { Several paragraphs of } \\
\text { different themes }\end{array}$ & $\begin{array}{l}\text { English to Hindi } \\
\text { language }\end{array}$ & $\begin{array}{l}\text { Bing Translator performed } \\
\text { better than Google } \\
\text { translator } \\
\text { while translating text, } \\
\text { English to Hindi } \\
\text { language or vice versa }\end{array}$ & Dhakar et al. (2013) \\
\hline
\end{tabular}

could examine whether psychometric properties are affected by different translation methods, could be of great importance, because response behaviors tends to be affected when respondents misread the critical words in reversely keyed items (Brauer and Proyer 2020). It would also be interesting to compare the actual time save by the hybrid method compared to the traditional method, which is not performed in present study. It will be interesting to report a statistical threshold value to which a hybrid method offered the advantage in terms of being cost-effective.

\section{Conclusions}

The present study suggests that the online translation method alone is not sufficient for the accurate translation of questionnaire and, therefore, we recommended that either traditional translation method or a hybrid method, which combines both online and traditional method, should be followed for the process of cross-cultural adaptation of self-report measures. In the first step, online translation has to be performed, and in second step proofreading and editing to be done traditionally by a language expert. By following such a hybrid method, the translation process could be carried out costeffectively within a short span of time with high accuracy and validity. 
Acknowledgments Authors highly acknowledged the expert committee for reviewing the present work. The manuscript was proofread by doc navigator@, Chandigarh.

Authors Contribution Dr. Jaimanti Bakshi and Dr. Dharam Vir conceptualized the work, Dr. Pankaj Aneja and Dr. Balwinder Singh did the forward translation, Dr. Meenu Gupta and Dr. Sumittar Singh Walia did the reverse translation. Dr. Rakesh Kapoor, Dr. Naresh K Panda and Dr. Krishna Soni helped in the validity testing. Atul K Goyal collected and compiled the raw data, generated the results, and drafted the manuscript.

Funding Information The work in this research is also supported by the Junior Research Fellowship (JRF) provided by ICMR, New Delhi wide file no. 3/13/JRF -2015/HRD.

\section{Compliance with Ethical Standards}

Present study has been approved by the institute ethics committee (Letter No. INT/IEC/2019/002539 with Ref No. NK/5657/PhD). Permission form the copyrighted owner of shame and stigma scale of head neck cancer, was obtained for translation of the instrument to Hindi language. Translation agreement was signed by all the translators.

Conflict of Interest The authors declare that the research was conducted in the absence of any commercial or financial relationships that could be construed as a potential conflict of interest.

\section{References}

Balk, E. M., Chung, M., Chen, M. L., Trikalinos, T. A., \& Chang, L. K. W. (2013). Assessing the accuracy of Google translate to allow data extraction from trials published in non-English languages. Agency for Healthcare Research and Quality, 12(13), 145.

Beaton, D. E., Bombardier, C., Guillemin, F., \& Ferraz, M. B. (2000). Guidelines for the process of crosscultural adaptation of self-report measures. Spine, 25(24), 3186-3191.

Bolarinwa, O. A. (2015). Principles and methods of validity and reliability testing of questionnaires used in social and health science researches. Nigerian Postgraduate Medical Journal, 22(4), 195.

Brauer, K., \& Proyer, R. T. (2020). Analyzing a German-language expanded form of the PhoPhiKat-45: Psychometric properties, factorial structure, measurement invariance with the Likert-version, and self-peer convergence. Journal of Personality Assessment, 1-11. https://doi.org/10.1080/00223891.2020.1720699.

Chen, X., Acosta, S., \& Barry, A. E. (2016). Evaluating the accuracy of Google translate for diabetes education material. JMIR diabetes, 1(1), e3.

Dhakar, B. S., Sinha, S. K., \& Pandey, K. K. (2013). A survey of translation quality of English to Hindi online translation systems (Google and Bing). Int J Sci Res Publ, 3(1), 1-4.

Goyal, A. K. (2015). Studies on phantom vibration and ringing syndrome among postgraduate students. Indian J Community Health, 27(1), 35-40.

Goyal, A. K., \& Saini, J. (2019). The phantom syndrome. Indian Journal of Social Psychiatry, 35(2), 102.

Guo, J.-W. (2016). Is Google translate adequate for facilitating instrument translation from English to mandarin? CIN: Computers, Informatics, Nursing, 34(9), 377-383.

Jones, T., Baxter, M., \& Khanduja, V. (2013). A quick guide to survey research. The Annals of The Royal College of Surgeons of England, 95(1), 5-7.

Kirchhoff, K., Turner, A. M., Axelrod, A., \& Saavedra, F. (2011). Application of statistical machine translation to public health information: A feasibility study. J Am Med Inform Assoc, 18(4), 473-478.

Kissane, D. W., Patel, S. G., Baser, R. E., Bell, R., Farberov, M., Ostroff, J. S., et al. (2013). Preliminary evaluation of the reliability and validity of the shame and stigma scale in head and neck cancer. Head \& neck, 35(2), 172-183.

Lau, A. S., Yusoff, M. S., Lee, Y.-Y., Choi, S.-B., Xiao, J.-Z., \& Liong, M.-T. (2018). Development and validation of a Chinese translated questionnaire: A single simultaneous tool for assessing gastrointestinal and upper respiratory tract related illnesses in pre-school children. Journal of Taibah University medical sciences, 13(2), 135-141. 
Minden, S. L., Feinstein, A., Kalb, R. C., Miller, D., Mohr, D. C., Patten, S. B., et al. (2014). Evidence-based guideline: Assessment and management of psychiatric disorders in individuals with MS: Report of the guideline development Subcommittee of the American Academy of Neurology. Neurology, 82(2), 174 181.

Mondal, H., Mondal, S., \& Mondal, S. (2019). Feasibility of using "Google translate” in adaptation of survey questionnaire from English to Bengali: A pilot study. Indian Journal of Social Psychiatry, 35(2), 119.

Pathak, A., \& Pakray, P. (2019). Neural machine translation for indian languages. J Intell Syst, 28(3), 465477.

Pirola, W. E., Paiva, B. S. R., Barroso, E. M., Kissane, D. W., Serrano, C. V. M. P., \& Paiva, C. E. (2017). Translation and cultural adaptation of the shame and stigma scale (SSS) into Portuguese (Brazil) to evaluate patients with head and neck cancer. Brazilian Journal of Otorhinolaryngology, 83(6), 697-704.

Prakash, S., \& Pallepati, A. (2016). Cross-cultural content validation of a modified service quality questionnaire in Kannada. Journal of Indian Association of Public Health Dentistry, 14(2), 171.

Rodrigues, I. B., Adachi, J. D., Beattie, K. A., \& MacDermid, J. C. (2017). Development and validation of a new tool to measure the facilitators, barriers and preferences to exercise in people with osteoporosis. $B M C$ Musculoskelet Disord, 18(1), 540.

Taylor, R. M., Crichton, N., Moult, B., \& Gibson, F. (2015). A prospective observational study of machine translation software to overcome the challenge of including ethnic diversity in healthcare research. Nursing Open, 2(1), 14-23.

Tsang, S., Royse, C. F., \& Terkawi, A. S. (2017). Guidelines for developing, translating, and validating a questionnaire in perioperative and pain medicine. Saudi Journal of Anaesthesia, 11(Suppl 1), S80.

Tseng, W.-T., Lee, Y., Hung, C.-F., Lin, P.-Y., Chien, C.-Y., Chuang, H.-C., et al. (2019). Validation of the Chinese version of the shame and stigma scale in patients with head and neck cancer. Cancer Management and Research, 11, 10297.

Vidhayasai, T., Keyuravong, S., \& Bunsom, T. (2015). Investigating the use of Google translate in" terms and conditions" in an Airline's official website: Errors and implications. PASAA: Journal of Language Teaching and Learning in Thailand, 49, 137-169.

Waltz, C. F., \& Bausell, B. R. (1981). Nursing research: design statistics and computer analysis: Davis FA. Yaghmaie, F. (2003). Content validity and its estimation. Journal of Medical Education, 3(1), 25-27.

Publisher's Note Springer Nature remains neutral with regard to jurisdictional claims in published maps and institutional affiliations. 


\title{
Affiliations
}

\section{Atul Kumar Goyal ${ }^{1} \cdot$ Jaimanti Bakshi ${ }^{1} \cdot$ Naresh K Panda $^{1} \cdot$ Rakesh Kapoor $^{2}$. Dharam Vir $^{1} \cdot$ Krishan Kumar $^{3}$. Pankaj Aneja ${ }^{4} \cdot$ Balwinder Singh $^{5} \cdot$ Meenu Gupta $^{6} \cdot$ Sumittar Singh Walia ${ }^{7}$}

\author{
Jaimanti Bakshi \\ drjayabakshi@ymail.com \\ Naresh K Panda \\ npanda59@yahoo.co.in \\ Rakesh Kapoor \\ drkapoor.r@gmail.com \\ Dharam Vir \\ vir486@gmail.com \\ Krishan Kumar \\ keshusony@rediffmail.com \\ Pankaj Aneja \\ drpankajaneja@ rediffmail.com \\ Balwinder Singh \\ drbalvendrasingh@gmail.com \\ Meenu Gupta \\ mun110275@gmail.com \\ Sumittar Singh Walia \\ shsumittarsinghwalia@gmail.com
}

Department of Otolaryngology and Head \& Neck Surgery (ENT), Post Graduate Institute of Medical Education and Research (PGIMER), Chandigarh 160012, India

2 Department of Radiotherapy, Post Graduate Institute of Medical Education and Research (PGIMER), Chandigarh 160012, India

3 Department of Psychiatry, Post Graduate Institute of Medical Education and Research (PGIMER), Chandigarh 160012, India

4 Post Graduate Institute of Medical Education and Research (PGIMER), Chandigarh 160012, India

5 Department of Hindi, DAV College, Jalandhar 144008, India

6 Department of English and Cultural Studies, Panjab University, Chandigarh 160014, India

7 Department of English, DAV College, Sector 10, Chandigarh 160010, India 